\title{
TWISTED TORUS BUNDLES OVER ARITHMETIC VARIETIES
}

\author{
MIN HO LEE
}

(Communicated by Roe Goodman)

\begin{abstract}
A twisted torus is a nilmanifold which is the quotient of a real Heisenberg group by a cocompact discrete subgroup. We construct fiber bundles over arithmetic varieties whose fibers are isomorphic to a twisted torus, and express the complex cohomology of such bundles over certain Riemann surfaces in terms of automorphic forms.
\end{abstract}

\section{INTRODUCTION}

Heisenberg groups are certain two-step nilpotent groups and they play an important role in quantum mechanics, harmonic analysis, and many other areas of mathematics (see e.g. [2], [3], [5], [10], [13]). A twisted torus is a nilmanifold which is the quotient of a real Heisenberg group by a cocompact discrete subgroup. In this paper we consider fiber bundles over arithmetic varieties whose fibers are isomorphic to a twisted torus.

Let $V$ be a vector space of dimension $2 m$ over $\mathbb{Q}$ and let $\beta$ be a nondegenerate alternating bilinear form on $V$. If $V(\mathbb{R})$ denotes the real vector space $V \otimes_{\mathbb{Q}} \mathbb{R}$, then the real Heisenberg group $H(\mathbb{R}, \beta)$ associated to $\beta$ is the product $V(\mathbb{R}) \times \mathbb{R}$ with multiplication given by

$$
\left(v_{1}, c_{1}\right) \cdot\left(v_{2}, c_{2}\right)=\left(v_{1}+v_{2}, c_{1}+c_{2}+\beta\left(v_{1}, v_{2}\right) / 2\right)
$$

for $\left(v_{1}, c_{1}\right),\left(v_{2}, c_{2}\right) \in V(\mathbb{R}) \times \mathbb{R}$. Let $S p(V(\mathbb{R}), \beta)$ be the symplectic group determined by the bilinear form $\beta$ on $V(\mathbb{R})$, and let $\mathscr{X}_{m}$ be the Siegel upper half-space on which $S p(V(\mathbb{R}), \beta)$ operates. We define a group structure on the product $S p(V(\mathbb{R}), \beta) \times V(\mathbb{R}) \times \mathbb{R}$ with multiplication given by

$$
\left(g_{1}, v_{1}, c_{1}\right) \cdot\left(g_{2}, v_{2}, c_{2}\right)=\left(g_{1} g_{2}, v_{1}+g_{1} v_{2}, c_{1}+c_{2}+\beta\left(v_{1}, g_{1} v_{2}\right) / 2\right)
$$

for all $\left(g_{1}, v_{1}, c_{1}\right),\left(g_{2}, v_{2}, c_{2}\right) \in S p(V(\mathbb{R}), \beta) \times V(\mathbb{R}) \times \mathbb{R}$. Then the group $S p(V(\mathbb{R}), \beta) \times V(\mathbb{R}) \times \mathbb{R}$ acts on $\mathscr{H}_{m} \times V(\mathbb{R}) \times \mathbb{R}$ by

$$
(g, v, c) \cdot(z, w, d)=(g z, v+g w, c+d+\beta(v, g w) / 2)
$$

for $(g, v, c) \in S p(V(\mathbb{R}), \beta) \times V(\mathbb{R}) \times \mathbb{R}$ and $(z, w, d) \in \mathscr{H}_{m} \times V(\mathbb{R}) \times \mathbb{R}$.

Let $G$ be a semisimple algebraic group defined over $\mathbb{Q}$, and let $K$ be a maximal compact subgroup of the semisimple Lie group $G(\mathbb{R})$. We assume

Received by the editors March 24, 1993.

1991 Mathematics Subject Classification. Primary 22E40, 14K10, 11 F12.

(C)1995 American Mathematical Society 
that the symmetric space $D=G(\mathbb{R}) / K$ has a $G(\mathbb{R})$-invariant complex structure. Let $\Gamma \subset G(\mathbb{Q})$ be a torsion-free cocompact arithmetic subgroup of $G$, and let $X=\Gamma \backslash D$ be the corresponding arithmetic variety. Let $L$ be a lattice in $V(\mathbb{R})$ such that $\beta(L, L) \subset \mathbb{Z}$, and let $\Gamma_{0}$ be a torsion-free subgroup of $S p(L, \beta)$ of finite index. Let $\rho: G(\mathbb{R}) \rightarrow S p(V(\mathbb{R}), \beta)$ be a symplectic representation of $G(\mathbb{R})$, and let $\tau: D \rightarrow \mathscr{H}_{m}$ be a holomorphic map such that $\rho(\Gamma) \subset \Gamma_{0}$ and

$$
\tau(g y)=\rho(g) \tau(y) \text { for all } g \in G(\mathbb{R}) \text { and } y \in D .
$$

Then the group $\Gamma_{0} \times L \times \mathbb{Z}$ operates on the space $\mathscr{H}_{m} \times V(\mathbb{R}) \times \mathbb{R}$ properly discontinuously. We denote by $W_{0}$ the quotient space $\Gamma_{0} \times L \times \mathbb{Z} \backslash \mathscr{H}_{m} \times V(\mathbb{R}) \times \mathbb{R}$. Then the natural projection $\mathscr{H}_{m} \times V(\mathbb{R}) \times \mathbb{R} \rightarrow \mathscr{H}_{m}$ induces a fiber bundle $\pi_{0}: W_{0} \rightarrow X_{0}$ over the complex manifold $X_{0}=\Gamma_{0} \backslash \mathscr{H}_{m}$. If $\tau_{X}: X \rightarrow X_{0}$ is the map induced by $\tau$, then we obtain a twisted torus bundle $\pi_{W}: W \rightarrow X$ by pulling back the bundle $\pi_{0}: W_{0} \rightarrow X_{0}$ by the map $\tau_{X}: X \rightarrow X_{0}$, and the fibers of $\pi_{W}$ are isomorphic to the twisted torus $H(\mathbb{Z}, \beta)_{L} \backslash H(\mathbb{R}, \beta)$, where $H(\mathbb{Z}, \beta)_{L}=L \times \mathbb{Z}$.

In this paper, we express the complex cohomology of $W$ in terms of certain automorphic forms with respect to $\Gamma$ when $X=\Gamma \backslash \mathscr{H}$ is a compact Riemann surface associated to a quaternion algebra.

\section{TWISTED TORUS BUNDLES}

In this section, we construct a twisted torus bundle $\pi: W \rightarrow X$ over an arithmetic variety $X=\Gamma \backslash D$ whose fibers are isomorphic to the quotient $H(\mathbb{Z}, \beta)_{L} \backslash H(\mathbb{R}, \beta)$ of the real Heisenberg group $H(\mathbb{R}, \beta)$ by a cocompact discrete subgroup $H(\mathbb{Z}, \beta)_{L}$.

First, we shall construct a universal bundle $\pi_{0}: W_{0} \rightarrow X_{0}$. Let $V$ be a $\mathbb{Q}$-vector space of dimension $2 n>0$, and let $\beta$ be a nondegenerate alternating bilinear form on $V$. Let $S p(V, \beta)$ be the symplectic group determined by $\beta$, i.e.,

$$
S p(V, \beta)=\{g \in G L(V) \mid \beta(g u, g v)=\beta(u, v) \text { for all } u, v \in V\} .
$$

Let $V(\mathbb{R})$ be the real vector space $V \otimes_{\mathbb{Q}} \mathbb{R}$, and let $S p(V(\mathbb{R}), \beta)$ be the corresponding symplectic group. We define a group structure on the product $S p(V(\mathbb{R}), \beta) \times V(\mathbb{R}) \times \mathbb{R}$ with multiplication given by

$$
\left(g_{1}, v_{1}, c_{1}\right) \cdot\left(g_{2}, v_{2}, c_{2}\right)=\left(g_{1} g_{2}, v_{1}+g_{1} v_{2}, c_{1}+c_{2}+\beta\left(v_{1}, g_{1} v_{2}\right) / 2\right)
$$

for all $\left(g_{1}, v_{1}, c_{1}\right),\left(g_{2}, v_{2}, c_{2}\right) \in S p(V(\mathbb{R}), \beta) \times V(\mathbb{R}) \times \mathbb{R}$. Let $\mathscr{H}_{m}$ be the Siegel upper half-space

$$
\begin{array}{r}
\mathscr{H}_{m}=\left\{J \in G L(V(\mathbb{R})) \mid J^{2}=-1, \quad \beta(x, J y)\right. \text { is a positive definite } \\
\text { symmetric bilinear form in } x, y \in V(\mathbb{R})\}
\end{array}
$$

on which $S p(V(\mathbb{R}), \beta)$ operates.

Lemma 2.1. The group $S p(V(\mathbb{R}), \beta) \times V(\mathbb{R}) \times \mathbb{R}$ operates on $\mathscr{H}_{m} \times V(\mathbb{R}) \times \mathbb{R}$ by

$$
(g, v, c) \cdot(z, w, d)=(g z, v+g w, c+d+\beta(v, g w) / 2)
$$

for $(g, v, c) \in S p(V(\mathbb{R}), \beta) \times V(\mathbb{R}) \times \mathbb{R}$ and $(z, w, d) \in \mathscr{X}_{m} \times V(\mathbb{R}) \times \mathbb{R}$. 
Proof. Let $\left(g_{1}, v_{1}, c_{1}\right),\left(g_{2}, v_{2}, c_{2}\right) \in S p(V(\mathbb{R}), \beta) \times V(\mathbb{R}) \times \mathbb{R}$ and $(z, w, d)$ $\in \mathscr{H}_{m} \times V(\mathbb{R}) \times \mathbb{R}$. Then we have

$$
\begin{aligned}
&\left(\left(g_{1}, v_{1}, c_{1}\right) \cdot\left(g_{2}, v_{2}, c_{2}\right)\right) \cdot(z, w, d) \\
&=\left(g_{1} g_{2}, v_{1}+g_{1} v_{2}, c_{1}+c_{2}+\beta\left(v_{1}, g_{1} v_{2}\right) / 2\right) \cdot(z, w, d) \\
&=\left(g_{1} g_{2} z, v_{1}+g_{1} v_{2}+g_{1} g_{2} w,\right. \\
&\left.c_{1}+c_{2}+\beta\left(v_{1}, g_{1} v_{2}\right) / 2+d+\beta\left(v_{1}+g_{1} v_{2}, g_{1} g_{2} w\right) / 2\right) \\
&=\left(g_{1} g_{2} z, v_{1}+g_{1} v_{2}+g_{1} g_{2} w,\right. \\
&\left.c_{1}+c_{2}+d+\left(\beta\left(v_{1}, g_{1} v_{2}\right)+\beta\left(v_{1}, g_{1} g_{2} w\right)+\beta\left(v_{2}, g_{2} w\right)\right) / 2\right) ;
\end{aligned}
$$

here we used the relation $\beta\left(g_{1} v_{2}, g_{1} g_{2} w\right)=\beta\left(v_{2}, g_{2} w\right)$. On the other hand we have

$$
\begin{aligned}
&\left(g_{1}, v_{1}, c_{1}\right) \cdot\left(\left(g_{2}, v_{2}, c_{2}\right) \cdot(z, w, d)\right) \\
&=\left(g_{1}, v_{1}, c_{1}\right)\left(g_{2} z, v_{2}+g_{2} w, c_{2}+d+\beta\left(v_{2}, g_{2} w\right) / 2\right) \\
&=\left(g_{1} g_{2} z, v_{1}+g_{1}\left(v_{2}+g_{2} w\right)\right. \\
&\left.c_{1}+c_{2}+d+\beta\left(v_{2}, g_{2} w\right) / 2+\beta\left(v_{1}, g_{1}\left(v_{2}+g_{2} w\right)\right) / 2\right) \\
&=\left(g_{1} g_{2} z, v_{1}+g_{1} v_{2}+g_{1} g_{2} w,\right. \\
&\left.c_{1}+c_{2}+d+\left(\beta\left(v_{2}, g_{2} w\right)+\beta\left(v_{1}, g_{1} v_{2}\right)+\beta\left(v_{1}, g_{1} g_{2} w\right)\right) / 2\right) .
\end{aligned}
$$

Hence the lemma follows.

Let $L$ be a lattice in $V(\mathbb{R})$ such that $\beta(L, L) \subset \mathbb{Z}$, and let $\Gamma_{0}$ be a torsionfree subgroup of $\operatorname{Sp}(L, \beta)$ of finite index, where

$$
S p(L, \beta)=\{g \in S p(V(\mathbb{R}), \beta) \mid g L=L\} .
$$

Then the group $\Gamma_{0} \times L \times \mathbb{Z}$ operates on the space $\mathscr{K}_{m} \times V(\mathbb{R}) \times \mathbb{R}$ properly discontinuously. We denote by $W_{0}$ the quotient space $\Gamma_{0} \times L \times \mathbb{Z} \backslash \mathscr{H}_{m} \times V(\mathbb{R}) \times \mathbb{R}$. Then the natural projection $\mathscr{U}_{m} \times V(\mathbb{R}) \times \mathbb{R} \rightarrow \mathscr{X}_{m}$ induces a fiber bundle $\pi_{W, 0}: W_{0} \rightarrow X_{0}$ over the complex manifold $X_{0}=\Gamma_{0} \backslash \mathscr{H}_{m}$.

Let $H(\mathbb{R}, \beta)$ denote the real Heisenberg group associated to $\beta$. Thus $H(\mathbb{R}, \beta)=V(\mathbb{R}) \times \mathbb{R}$ with its multiplication operation given by

$$
(v, c) \cdot(w, d)=(v+w, c+d+\beta(v, w) / 2) .
$$

Let $H(\mathbb{Z}, \beta)_{L}$ be the subgroup $L \times \mathbb{Z} \subset V(\mathbb{R}) \times \mathbb{R}$ of $H(\mathbb{R}, \beta)$. Then each fiber of $\pi_{0}$ is isomorphic to the quotient $H(\mathbb{Z}, \beta)_{L} \backslash H(\mathbb{R}, \beta)$, which is a circle bundle over a torus called a twisted torus.

Let $G$ be a semisimple algebraic group defined over $\mathbb{Q}$, and let $K$ be a maximal compact subgroup of the semisimple Lie group $G(\mathbb{R})$. We assume that the symmetric space $D=G(\mathbb{R}) / K$ has a $G(\mathbb{R})$-invariant complex structure. Let $\Gamma \subset G(\mathbb{Q})$ be a torsion-free cocompact arithmetic subgroup $G$. Then the quotient $X=\Gamma \backslash D$ has a structure of a complex projective variety called an 
arithmetic variety (see e.g. [6]). Let $\rho: G \rightarrow S p(V, \beta)$ be a symplectic representation and $\tau: D \rightarrow \mathscr{H}_{m}$ a holomorphic map such that $\rho(\Gamma) \subset \Gamma_{0}$ and

$$
\tau(g y)=\rho(g) \tau(y) \text { for all } g \in G(\mathbb{R}) \text { and } y \in D .
$$

If $\tau_{X}: X \rightarrow X_{0}$ is the map induced by $\tau$, then we obtain a map $\pi_{W}: W \rightarrow X$ by pulling back the bundle $W_{0}$ via the map $\tau_{X}: X \rightarrow X_{0}$. Thus we have the following commutative diagram:

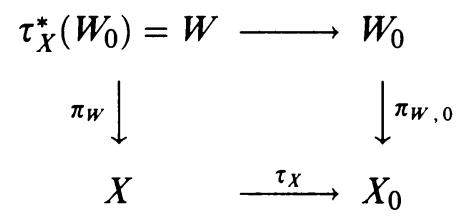

The map $\pi_{W}: W \rightarrow X$ is a fiber bundle, called a twisted torus bundle, and each of its fibers is isomorphic to the twisted torus $H(\mathbb{Z}, \beta)_{L} \backslash H(\mathbb{R}, \beta)$. This bundle can also be described as follows: Let $W=\Gamma \times L \times \mathbb{Z} \backslash D \times V(\mathbb{R}) \times \mathbb{R}$, where $\Gamma \times L \times \mathbb{Z}$ acts on $D \times V(\mathbb{R}) \times \mathbb{R}$ by

$$
(\gamma, l, c) \cdot(z, w, d)=(\rho(\gamma) z, l+\rho(\gamma) w, c+d+\beta(l, \rho(g) w) / 2)
$$

for all $(\gamma, l, c) \in \Gamma \times L \times \mathbb{Z}$ and $(z, w, d) \in D \times V(\mathbb{R}) \times \mathbb{R}$. Then the twisted torus bundle $\pi_{W}: W \rightarrow X$ is the fiber bundle induced by the natural projection

$$
\Gamma \times L \times \mathbb{Z} \backslash D \times V(\mathbb{R}) \times \mathbb{R} \rightarrow \Gamma \backslash D=X .
$$

\section{KUGA FIBER VARIETIES}

In this section, we review the construction of Kuga fiber varieties over arithmetic varieties whose fibers are polarized abelian varieties. Let $V(\mathbb{R}), \beta, L$, $\mathscr{H}_{m}$ and $\Gamma_{0}$ as in $\S 2$. Then each element $J \in \mathscr{H}_{m}$ defines a complex structure on $V(\mathbb{R})$ and there is a unique complex analytic structure on $\mathscr{H}_{m} \times V(\mathbb{R})$ such that the projection $P: \mathscr{H}_{m} \times V(\mathbb{R}) \rightarrow \mathscr{H}_{m}$ becomes a complex vector bundle over $\mathscr{H}_{m}$. For each $J$, if we denote the complex vector space $(V(\mathbb{R}), J)$ by $V(\mathbb{R})_{J}$, then the complex torus $Y_{J}=V(\mathbb{R})_{J} / L$ is an abelian variety that has a polarization determined by $\beta$. We set

$$
Y_{\mathscr{X}_{m}}=L \backslash \mathscr{H}_{m} \times V(\mathbb{R}),
$$

where the action of $L$ on $\mathscr{H}_{m} \times V(\mathbb{R})$ is given by

$$
l \cdot(J, v)=(J, v+l) \text { for } J \in \mathscr{H}_{m}, v \in V(\mathbb{R}) \text { and } l \in L .
$$

Then the vector bundle $P: \mathscr{H}_{m} \times V(\mathbb{R}) \rightarrow \mathscr{H}_{m}$ induces the fiber bundle $\pi_{\mathscr{H}_{m}}$ : $Y_{\mathscr{X}_{m}} \rightarrow \mathscr{H}_{m}$ whose fibers are abelian varieties polarized by $\beta$. Then the quotient $X_{0}=\Gamma_{0} \backslash \mathscr{H}_{m}$ is an arithmetic variety that can be considered as a Zariski open subset of a complex projective variety. Now the fiber bundle $\pi_{\mathscr{L}_{m}}$ : $Y_{\mathscr{X}_{m}} \rightarrow \mathscr{X}_{m}$ induces a universal family of abelian varieties $\pi_{Y, 0}: Y_{0} \rightarrow X_{0}$ over $X_{0}$ described in Example 1 in [11, §IV.7].

Let $G, K, D=G(\mathbb{R}) / K, \Gamma \subset G(\mathbb{Q}), X=\Gamma \backslash D, \rho: G(\mathbb{R}) \rightarrow S p(V(\mathbb{R}), \beta)$ and $\tau: D \rightarrow \mathscr{H}_{m}$ be as in $\S 2$. If $\tau_{X}: X \rightarrow X_{0}$ is the map induced by $\tau: D \rightarrow$ $\mathscr{H}_{m}$, then by pulling back the bundle $\pi_{Y, 0}: Y_{0} \rightarrow X_{0}$ via the map $\tau_{X}$ we obtain the Kuga fiber variety $\pi_{Y}: Y \rightarrow X$ over the arithmetic variety $X=\Gamma \backslash D$. Kuga fiber varieties can also be described as follows: The semidirect product $\Gamma \ltimes_{\rho} L$ 
with respect to the representation $\rho: \Gamma \rightarrow \operatorname{Aut}(L)$ operates on the product manifold $D \times V(\mathbb{R})$ properly discontinuously by

$$
(\gamma, l) \cdot(y, v)=(\gamma y, \rho(\gamma) v+l)
$$

for $(\gamma, l) \in \Gamma \times_{\rho} L$ and $(y, v) \in D \times V(\mathbb{R})$, and this action coincides with the restriction of the operation of $\Gamma \times L \times \mathbb{Z}$ on $D \times V(\mathbb{R}) \times \mathbb{R}$ described at the end of $\S 2$ to the group $\Gamma \times L \subset \Gamma \times L \times \mathbb{Z}$. We set

$$
Y=\Gamma \ltimes_{\rho} L \backslash D \times V(\mathbb{R}),
$$

and denote by $\pi$ the natural projection of $Y$ onto $X=\Gamma \backslash D$. It is known that $Y$ has a structure of a complex projective variety and that the fiber $Y_{x}$ over each $x \in X$ is an abelian variety isomorphic to the quotient $V(\mathbb{R}) / L$ polarized by $\beta$ (see [7], [8], [9], [11, Chapter 4] for details).

\section{THE COHOMOLOGY}

Let $\pi_{W}: W \rightarrow X$ be the fiber bundle constructed in $\S 2$ and let $\pi_{Y}: Y \rightarrow X$ be the Kuga fiber variety as in $\S 2$. Since the restriction of the operation of $\Gamma \times L \times \mathbb{Z}$ on $D \times V(\mathbb{R}) \times \mathbb{R}$ described in $\S 2$ to $\Gamma \times L$ coincides with the action of $\Gamma \times_{\rho} L$ on $D \times V(\mathbb{R})$ in $\S 3$, the natural projection $\mathscr{H}_{m} \times V(\mathbb{R}) \times \mathbb{R} \rightarrow \mathscr{H}_{m} \times V(\mathbb{R})$ induces a fiber bundle $\pi_{W, Y}: W \rightarrow Y$ whose fibers are isomorphic to the circle $\mathbb{R} / \mathbb{Z}$. Thus $W$ can be considered as a circle bundle over the Kuga fiber variety $Y$. In this section, we express the complex cohomology $H^{*}(W, \mathbb{C})$ of the twisted torus bundle $W$ in terms of the cohomology $H^{*}(Y, \mathbb{C})$ of the Kuga fiber variety $Y$.

Theorem 4.1(Hochschild-Serre). For any group extension

$$
1 \rightarrow \Phi \rightarrow \mathfrak{G} \rightarrow \Delta \rightarrow 1
$$

and any $\mathfrak{G}$-module $\mathscr{M}$, there is a spectral sequence $\left\{\mathscr{E}_{r}\right\}$ with $\mathscr{E}_{2}$-term

$$
\mathscr{E}_{2}^{p q}=H^{p}\left(\Delta, H^{q}(\Phi, \mathscr{M})\right)
$$

that converges to the cohomology $H^{*}(\mathfrak{G}, \mathscr{M})$.

Proof. See [4] or [1, §VII.6].

Now we apply the Hochschild-Serre theorem to the groups $\mathfrak{G}=\Gamma \times L \times \mathbb{Z}$, $\Delta=\Gamma \times L$, the abelian group $\Phi=\mathbb{Z}$, and the trivial $\Gamma \times L \times \mathbb{Z}$-module $\mathscr{M}=\mathbb{C}$. Thus we have a spectral sequence $\left\{E_{r}\right\}$ with

$$
E_{2}^{p, q}=H^{p}\left(\Gamma \times L, H^{q}(\mathbb{Z}, \mathbb{C})\right)
$$

and

$$
H^{r}(\Gamma \times L \times \mathbb{Z}, \mathbb{C}) \cong \bigoplus_{p+q=r} E_{\infty}^{p, q} .
$$

Lemma 4.2. The cohomology $H^{q}(\mathbb{Z}, \mathbb{C})$ of the abelian group $\mathbb{Z}$ with coefficients in the trivial $\mathbb{Z}$-module $\mathbb{C}$ is given as follows:

$$
H^{0}(\mathbb{Z}, \mathbb{C})=H^{1}(\mathbb{Z}, \mathbb{C})=\mathbb{C}, \quad H^{q}(\mathbb{Z}, \mathbb{C})=0 \text { for } q \neq 0,1 .
$$

Proof. The homology $H_{1}(\mathbb{Z}, \mathbb{Z})$ of the group $\mathbb{Z}$ with coefficients in the trivial $\mathbb{Z}$-module $\mathbb{Z}$ is isomorphic to $\mathbb{Z} /[\mathbb{Z}, \mathbb{Z}]=\mathbb{Z}$. Hence we have

$$
H_{1}(\mathbb{Z}, \mathbb{C})=\mathbb{Z} \otimes \mathbb{C}=\mathbb{C}, \quad H^{1}(\mathbb{Z}, \mathbb{C})=\mathbb{C}^{*}
$$


and

$$
H^{q}(\mathbb{Z}, \mathbb{C})=\bigwedge^{q}\left(H^{1}(\mathbb{Z}, \mathbb{C})\right)=\bigwedge^{q}\left(\mathbb{C}^{*}\right),
$$

where $\mathbb{C}^{*}$ denotes the dual of the trivial $\mathbb{Z}$-module $\mathbb{C}$. Since $\Lambda^{q}\left(\mathbb{C}^{*}\right)=\mathbb{C}$ for $q=0,1$ and $\Lambda^{q}\left(\mathbb{C}^{*}\right)=0$ for $q \neq 0,1$, the proof is complete.

From the relation $H^{q}(\mathbb{Z}, \mathbb{C})=\Lambda^{q}\left(\mathbb{C}^{*}\right)$ given in the proof of Lemma 4.2, it follows that the spectral sequence $\left\{E_{r}\right\}$ has the $E_{2}$-term

$$
E_{2}^{p, q}=H^{p}\left(\Gamma \times L, H^{p}(\mathbb{Z}, \mathbb{C})\right)=H^{q}\left(\Gamma \times L, \bigwedge^{q}\left(\mathbb{C}^{*}\right)\right) .
$$

Proposition 4.3. The spectral sequence $\left\{E_{r}\right\}$ degenerates at $E_{2}$.

Proof. For each positive integer $a$, the map $\mu_{a}: \Gamma \times L \times \mathbb{Z} \rightarrow \Gamma \times L \times \mathbb{Z}$ defined by

$$
\mu_{a}(\gamma, l, k)=\left(\gamma, a l, a^{2} k\right)
$$

for $(\gamma, l, k) \in \Gamma \times L \times \mathbb{Z}$ is a homomorphism of groups. Hence $\mu_{a}$ induces maps

$$
\mu_{a}^{*}: H^{r}(\Gamma \times L \times \mathbb{Z}, \mathbb{C}) \rightarrow H^{r}(\Gamma \times L \times \mathbb{Z}, \mathbb{C})
$$

and $\left(\mu_{a}^{*}\right)_{r}^{p, q}: E_{r}^{p, q} \rightarrow E_{r}^{p, q}$ such that

$$
d_{r} \circ\left(\mu_{a}^{*}\right)_{r}^{p, q}=\left(\mu_{a}^{*}\right)_{r}^{p+r, q-r+1} \circ d_{r},
$$

where $d_{r}: E_{r}^{p, q} \rightarrow E_{r}^{p+r, q-r+1}$ is the boundary map. Since $E_{2}^{p, q}=$ $H^{q}\left(\Gamma \times L, \Lambda^{q}\left(\mathbb{C}^{*}\right)\right)$, the map $\left(\mu_{a}^{*}\right)_{2}^{p, q}$ can be considered as the multiplication by $a^{2 q}$. Hence we have

$$
d_{2}\left(a^{2 q} v\right)=a^{2 q-2}\left(d_{2}(v)\right)
$$

for all $v \in E_{2}^{p, q}$. Since $a$ is an arbitrary positive integer, it follows that $d_{2}=0$. Similarly, we obtain $d_{s}=0$ for all $s \geq 2$. Hence $\left\{E_{r}\right\}$ degenerates at $E_{2}$ and the proof is complete.

Theorem 4.4. The cohomology $H^{r}(Y, \mathbb{C})$ with coefficient $\mathbb{C}$ has the decomposition

$$
H^{r}(W, \mathbb{C})=H^{r}(Y, \mathbb{C}) \oplus H^{r-1}(Y, \mathbb{C})
$$

for $1 \leq r \leq \operatorname{dim}_{\mathbb{R}} W$.

Proof. Since we have

$$
W=\Gamma \times L \times \mathbb{Z} \backslash D \times V(\mathbb{R}) \times \mathbb{R} \text { and } Y=\Gamma \times L \backslash D \times V(\mathbb{R}),
$$

the group cohomologies $H^{r}(\Gamma \times L \times \mathbb{Z}, \mathbb{C})$ and $H^{r}(\Gamma \times L, \mathbb{C})$ with coefficients in the trivial module $\mathbb{C}$ can be identified with the complex cohomologies $H^{r}(W, \mathbb{C})$ and $H^{r}(Y, \mathbb{C})$ respectively. Using Proposition 4.3 and Lemma 4.2, we have

$$
\begin{aligned}
H^{r}(W, \mathbb{C}) & =H^{r}(\Gamma \times L \times \mathbb{Z}, \mathbb{C})=\bigoplus_{p+q=r} E_{2}^{p, q}=\bigoplus_{p+q=r} H^{p}\left(\Gamma \times L, \bigwedge^{q}\left(\mathbb{C}^{*}\right)\right) \\
& =H^{r}(\Gamma \times L, \mathbb{C}) \oplus H^{r-1}(\Gamma \times L, \mathbb{C})=H^{r}(Y, \mathbb{C}) \oplus H^{r-1}(Y, \mathbb{C}) .
\end{aligned}
$$

Hence the theorem follows. 


\section{AUTOMORPHIC FORMS}

In this section we consider twisted torus bundles and Kuga fiber varieties of a special type. Let $G$ be the semisimple algebraic $\mathbb{Q}$-group $S L_{2}$ so that $G(\mathbb{R})=S L(2, \mathbb{R}), K=S O(2)$, and $D$ is the Poincare upper half-plane $\mathscr{H}$. Let $B$ be an indefinite quaternion algebra over $\mathbb{Q}$ such that $B \otimes_{\mathbb{Q}} \mathbb{R}=M_{2}(\mathbb{R})$, and let $\odot$ be a maximal order in $B$. Given a positive integer $N$, let $\Gamma_{N}$ be the discrete subgroup of $S L(2, \mathbb{R})$ defined by

$$
\Gamma_{N}=\{\alpha \in \mathscr{O} \mid \alpha \mathscr{O}=\mathscr{O}, \quad \operatorname{det} \alpha=1, \quad \alpha=1(\bmod N \mathscr{O})\} .
$$

Then the quotient space $\Gamma_{N} \backslash \mathscr{H}$ is a compact Riemann surface. We set

$$
V_{m}=M(2, \mathbb{R})^{m}, \quad L_{m}=\mathscr{O}^{m},
$$

and define the representation $\rho_{m}: G(\mathbb{R}) \rightarrow G L\left(V_{m}\right)$ by

$$
\rho_{m}(g)\left(\mu_{1}, \ldots, \mu_{m}\right)=\left(g \mu_{1}, \ldots, g \mu_{m}\right)
$$

for all $\left(\mu_{1}, \ldots, \mu_{m}\right) \in M(2, \mathbb{R})^{m}$. Let the twisted torus bundle $\pi_{W}: W \rightarrow X$ and the Kuga fiber variety $\pi_{Y}: Y \rightarrow X$ be the ones constructed from $D=\mathscr{C}$, $\Gamma=\Gamma_{N}, L=L_{m}$ and $\rho=\rho_{m}$.

Since $X=\Gamma \backslash \mathscr{H}=\Gamma_{N} \backslash \mathscr{H}$ is compact, the group $\Gamma$ has no cusps in $\mathscr{H}$; hence any holomorphic function $f: \mathscr{H} \rightarrow \mathbb{C}$ satisfying

$$
f(\gamma z)=(c z+d)^{k} F(z) \text { for } \quad z \in \mathscr{H}, \quad \gamma \in\left(\begin{array}{ll}
* & * \\
c & d
\end{array}\right) \in \Gamma \subset S L(2, \mathbb{R})
$$

is both an automorphic form and a cusp form of weight $k$ with respect to $\Gamma$ (see e.g. [12]). We denote by $S_{k}(\Gamma)$ the space of all automorphic forms of weight $k$ with respect to $\Gamma$.

Theorem 5.1. Let $\pi_{W}: W \rightarrow X$ be the twisted torus bundle over $X=\Gamma \backslash \mathscr{H}$ associated to an indefinite quaternion algebra $B$ as described above, and let $\omega_{0}$ be the cohomology class of the two-form $y^{-2}(d z \wedge d \bar{z})$ in $H^{2}(W, \mathbb{C})$. Then, for each $r \geq 3$, the complex cohomology $H^{r}(W, \mathbb{C})$ of $W$ has the following decomposition:

$$
\begin{aligned}
H^{r}(W, \mathbb{C})= & A_{r}\left(S_{r+1}(\Gamma) \oplus \overline{S_{r+1}(\Gamma)}\right) \\
& \oplus\left(\bigoplus_{j=0}^{r-2} B_{r, j}\left(S_{j+2}(\Gamma) \oplus \overline{S_{j+2}(\Gamma)}\right)\right) \oplus C_{r} \mathbb{C} \oplus C_{r-2} \mathbb{C} \omega_{0},
\end{aligned}
$$

where $A_{r}=\left(\begin{array}{c}2 m \\ r-1\end{array}\right)$,

$$
\boldsymbol{B}_{r, j}=\left\{\begin{array}{l}
\left(\begin{array}{c}
2 m \\
r-j_{0}-1
\end{array}\right)\left(\begin{array}{c}
2 m \\
j_{0}-1
\end{array}\right)-\left(\begin{array}{c}
2 m \\
r-j_{0}
\end{array}\right)\left(\begin{array}{c}
2 m \\
j_{0}-2
\end{array}\right) \quad \text { if } j=r-2 j_{0}, \\
\left(\begin{array}{c}
2 m \\
r-j_{0}-1
\end{array}\right)\left(\begin{array}{c}
2 m \\
j_{0}
\end{array}\right)-\left(\begin{array}{c}
2 m \\
r-j_{0}
\end{array}\right)\left(\begin{array}{c}
2 m \\
j_{0}-1
\end{array}\right) \quad \text { if } j=r-2 j_{0}-1,
\end{array}\right.
$$

and

$$
C_{r}=\left(\begin{array}{c}
2 m \\
r_{0}
\end{array}\right)^{2}-\left(\begin{array}{c}
2 m \\
r_{0}+1
\end{array}\right)\left(\begin{array}{c}
2 m \\
r_{0}-1
\end{array}\right) \text { if } r=2 r_{0} \text { or } 2 r_{0}+1
$$


Proof. By Equation (11) in [7, §IV.2] or Equation (1) in [8] we have a decomposition of the cohomology $H^{p}(Y, \mathbb{C})$ of the Kuga fiber variety $Y$ of the form

$H^{p}(Y, \mathbb{C})=a(p, 0) \mathbb{C} \oplus\left(\bigoplus_{j=0}^{p-1} a(p-1, j)\left(S_{j+2}(\Gamma) \oplus \overline{S_{j+2}(\Gamma)}\right)\right) \oplus a(p-2,0) \mathbb{C} \omega_{0}$,

where

$$
a(s, t)=\left(\begin{array}{c}
2 m \\
(s+t) / 2
\end{array}\right)\left(\begin{array}{c}
2 m \\
(s-t) / 2
\end{array}\right)-\left(\begin{array}{c}
2 m \\
(s+t) / 2+1
\end{array}\right)\left(\begin{array}{c}
2 m \\
(s-t) / 2-1
\end{array}\right)
$$

if $s \equiv t(\bmod 2)$, and $a(s, t)=0$ if $s \not \equiv t(\bmod 2)$. Using Theorem 4.4, we have

$$
\begin{aligned}
H^{r}(W, \mathbb{C})= & (a(r, 0)+a(r-1,0)) \mathbb{C} \\
& \oplus\left(\bigoplus_{j=0}^{r-2}(a(r-1, j)+a(r-2, j))\left(S_{j+2}(\Gamma) \oplus \overline{S_{j+2}(\Gamma)}\right)\right) \\
& \oplus a(r-1, r-1)\left(S_{r+1}(\Gamma) \oplus \overline{S_{r+1}(\Gamma)}\right) \\
& \oplus(a(r-2,0)+a(r-3,0)) \mathbb{C} \omega_{0} .
\end{aligned}
$$

The formula for $A_{r}$ follows from $a(r-1, r-1)=\left(\begin{array}{c}2 m \\ r-1\end{array}\right)$. The formula for $B_{r, j}$ is obtained from the relations

$a(r-1, j)+a(r-2, j)=\left\{\begin{array}{l}a(r-2, j)=a\left(r-2, r-2 j_{0}\right) \quad \text { if } j=2-2 j_{0}, \\ a(r-1, j)=a\left(r-1, r-2 j_{0}-1\right) \\ \text { if } j=r-2 j_{0}-1 .\end{array}\right.$

For $C_{r}$, we have $a(r, 0)+a(r-1,0)=a(r, 0)=a\left(2 r_{0}, 0\right)$ if $r=2 r_{0}$, and $a(r, 0)+a(r-1,0)=a(r-1,0)=a\left(2 r_{0}, 0\right)$ if $r=2 r_{0}+1$. Hence we obtain the formula for $C_{r}$, and the proof is complete.

\section{REFERENCES}

1. K. Brown, Cohomology of groups, Springer-Verlag, New York, 1982.

2. P. Cartier, Quantum mechanical commutation relations and theta functions, Proc. Sympos. Pure Math., vol. 9, Amer. Math. Soc., Providence, RI, 1966, pp. 361-386.

3. G. Folland, Harmonic analysis in phase space, Princeton Univ. Press, Princeton, NJ, 1989.

4. G. Hochschild and J.-P. Serre, Cohomology of group extensions, Trans. Amer. Math. Soc. 74 (1953), 110-134.

5. R. Howe, On the role of the Heisenberg group in harmonic analysis, Bull. Amer. Math. Soc. (N. S.) 3 (1980), 821-843.

6. D. Kazhdan, On arithmetic varieties, Lie Groups and Their Representations, Halsted, Budapest, 1971, pp. 151-217.

7. M. Kuga, Fiber varieties over a symmetric space whose fibers are abelian varieties I, II, lecture notes, Univ. Chicago, 1963/64.

8. ___ Fiber varieties over a symmetric space whose fibers are abelian varieties, Proc. Sympos. Pure Math., vol. 9, Amer. Math. Soc., Providence, RI, 1966, pp. 338-346.

9. M. H. Lee, Conjugates of equivariant holomorphic maps of symmetric domains, Pacific J. Math. 149 (1991), 127-144. 
10. D. Mumford, Tata lectures on theta III, Birkhäuser, Boston, MA, 1991.

11. I. Satake, Algebraic structures of symmetric domains, Princeton Univ. Press, Princeton, NJ, 1980.

12. G. Shimura, Arithmetic theory of automorphic functions, Princeton Univ. Press, Princeton, NJ, 1971.

13. M. Taylor, Noncommutative harmionic analysis, Amer. Math. Soc., Providence, RI, 1986.

Department of Mathematics, University of Northern Iowa, Cedar Falls, Iowa 50614 E-mail address: lee@math.uni.edu 